\title{
Étude de la ration alimentaire des chevaux d'une société hippique à Saïgon
}

\author{
par C. RICHARD
}

A la suite d'une maladie parasitaire du type Surra qui provoqua la mort de plusieurs chevaux de l'équipage d'une société hippique à Saïgon, on nous demanda d'examiner la valeur nutritive et énergétique de leurs rations. Une nourriture non équilibrée et carencée en certains principes alimentaires indispensables peut en effet prédisposer des animaux apparemment sains à l'infection et à la maladie.

Les chevaux de selle de cette société hippique au nombre d'une trentaine, âgés de 9 à 20 ans, d'un poids moyen de 450 kilogrammes, appartiennent à la race des pur-sang anglais ou correspondent à des demi-sang australiens.

II est à noter qu'ils retrouvèrent dans l'ensemble une bonne santé et qu'ils purent effectuer normalement leur entraînement quotidien après un traitement à base de Moranyl 309 Poulenc, trypanocide à élimination lente, de stovarsol, parasiticide arsenical pentavalent et de vitamines du groupe $B$ (vitamines $B_{1}$ et $B_{12}$ ).

\section{Constituants de la ration alimentaire lors de l'enquête}

Au moment où nous avons procédé à cette enquête, la ration journalière de chaque cheval se composait de :

le matin,

- 3 litres de paddy germé, soil 1.650 gram mes

- 1 litre de mais, soit 600 grammes

- Mélasse de canne à sucre : 350 grammes (ce poids correspond aux $3.4 \mathrm{du}$ volume d'une boîte standard de lait concentré sucré)

le soir,

- 3 litres de riz corgo rouge, soit 3.500 grammes

Reçu pour publication : jan jier 1961.

Rev. Elev. Méd. vét. Pays trop., 1961, 14, no 1.
- Sel ordinaire : 20 grammes

entre les 2 repas et principalement le soir,

- Herbe verte : 5 kilogrammes

- Eau : ad libitum.

Composition chimique des aliments ingérés Apports nutritifs et énergétiques par jour et par cheval

Pour établir la composition chimique centésimale de chacun des aliments énumérés ci-dessus, nous avons uti!isé les techniques analytiques proposées par A. VIALARD GOUDOU dans son étude sur la « Composition ef la valeur nutritive de quelques aliments du Sud-Vietnam et de l'Asic tropicale » (8), sauf en ce qui concerne le potassium et le sodium, dosés respectivement par gravimétrie du tétraphénylborure de potassium (5) et par gravimétrie de l'acétate triple de sodium-zinc-uranyle, après élimination des phosphates à l'état de phosphate ammoniacomagnésien (6).

Nous avons ainsi successivement dosé l'humidité, les lipides, les protides, les glucides, la cellulose, les cendres minérales, la silice $\left(\mathrm{SiO}_{2}\right)$, les ions calcium ( $\mathrm{Ca}$ ), phosphore $(\mathrm{P})$, fer $(\mathrm{Fe})$, potassium $(K)$ et sodium $(\mathrm{Na})$ dans chacun des 6 aliments constituant la dite ration, à savoir poddy germé, maîs, mélasse de canne, riz cargo rouge, herbe verte et sel ordinaire.

Les valeurs énergétiques correspondantes ont été calculées en utilisant les coefficients de transformation calorifique de Atwater, soit 4 calories par gramme de protide ou de glucide et 9 calories por gramme de lipide, et rapportées à 100 grammes.

Par ailleurs, comme le pouvoir calorifique des aliments à ufilisation vétérinaire est souvent indiqué en unités fourragères (par abréviation U. F.), nous avons également employé cé mode d'expression. Selon la méthode danoise des unités fourragères « Un kilogramme de farine 
TABLAAJ I - Table de composition des 6 aliments constituant la ration \& riz cargo rouge, maïs, paddy germé, mélasse de canne, herbe verte et sel.

(Résultats rapportés à 100 grames d'aliment).

\begin{tabular}{|c|c|c|c|c|c|c|c|}
\hline & & $\begin{array}{l}\text { Riz cargo } \\
\text { rouge }\end{array}$ & Mairs & Paddy germe & $\begin{array}{c}\text { Mélasse de } \\
\text { canne }\end{array}$ & Ferbe verte & Sel \\
\hline \multicolumn{2}{|c|}{ Fumidité } & $20,55 \mathrm{~g}$ & $15,86 \mathrm{~g}$ & $42,01 \mathrm{~g}$ & $23,66 \mathrm{~g}$ & $63,89 \mathrm{~B}$ & $4,57 \mathrm{~B}$ \\
\hline \multicolumn{2}{|c|}{ Lipices } & $1,91 \mathrm{~g}$ & $5,96 \mathrm{~g}$ & $1,23 \mathrm{~g}$ & - & $0,56 \mathrm{E}$ & - \\
\hline \multicolumn{2}{|c|}{ Protides } & $6,49 \mathrm{~g}$ & $8,93 \mathrm{~g}$ & $4,43.8$ & $1,37 \mathrm{E}$ & 4,31 B & - \\
\hline \multicolumn{2}{|c|}{ Glucides } & $66,24 \mathrm{~g}$ & $59,58 \mathrm{~g}$ & $34,87 \mathrm{~g}$ & $66,15 \cdot g$ & 8,918 & - \\
\hline \multicolumn{2}{|c|}{ Ceilulose } & $3,61 \mathrm{~g}$ & $7,93 \mathrm{~g}$ & $14,60 \mathrm{~g}$ & - & $18,54 \mathrm{E}$ & - \\
\hline \multicolumn{2}{|c|}{ Cendres à $550^{\circ}$} & $1,20 \mathrm{~g}$ & $1,74 \mathrm{~g}$ & $2,93 \mathrm{~g}$ & $5,18 \mathrm{~g}$ & $3,78 \mathrm{E}$ & $94,55 \mathrm{~B}$ \\
\hline \multicolumn{2}{|c|}{ Silice } & $116 \mathrm{mg}$ & $74 \mathrm{mg}$ & $2250 \mathrm{mg}$ & $287 \mathrm{mg}$ & $1643 \mathrm{mg}$ & - \\
\hline \multicolumn{2}{|c|}{ Carcium } & $13,52 \mathrm{mg}$ & $12,48 \mathrm{mg}$ & $19,60 \mathrm{~g}$ & $859,50 \mathrm{mg}$ & $265 \mathrm{mg}$ & $200 \mathrm{me}$ \\
\hline \multicolumn{2}{|c|}{ Phosphore } & $245 \mathrm{mg}$ & $350 \mathrm{mg}$ & $116,50 \mathrm{mg}$ & $30 \mathrm{mg}$ & $98 \mathrm{mg}$ & 0 \\
\hline Rapport & $\mathrm{Ca} / \mathrm{p}$ & 0,05 & 0,03 & 0,16 & 28,65 & 2,69 & - \\
\hline \multicolumn{2}{|c|}{ Potassium } & $177 \mathrm{mg}$ & $340 \mathrm{mg}$ & $106 \mathrm{~ms}$ & $1500 \mathrm{mg}$ & $565 \mathrm{mg}$ & 0 \\
\hline \multicolumn{2}{|c|}{ Sodium } & $30,8 \mathrm{Ig}$ & $40 \mathrm{mg}$ & $15 m$ & $164,1 \mathrm{mg}$ & 32 mE & $34,05 \mathrm{~g}$ \\
\hline Rapport & $\mathrm{K} / \mathrm{Na}$ & 5,74 & 8,50 & 7,06 & 9,14 & 17,65 & \\
\hline \multicolumn{2}{|c|}{ Fer } & $2,6 \mathrm{mg}$ & $3,6 \mathrm{mg}$ & $5,6 \mathrm{mg}$ & 17,2 mg & $10,3 \mathrm{mg}$ & - \\
\hline \multicolumn{2}{|c|}{$\begin{array}{l}\text { Calories } \\
\text { dues aux lipides }\end{array}$} & 17,19 & 53,64 & 11,07 & - & 5 & - \\
\hline \multicolumn{2}{|c|}{$\begin{array}{l}\text { Calories } \\
\text { dues anx protides }\end{array}$} & 25,96 & 35,72 & 17,36 & 5,48 & 17,20 & - \\
\hline \multicolumn{2}{|c|}{$\begin{array}{c}\text { Calories } \\
\text { dues aux glucides }\end{array}$} & 264,96 & 238,32 & 139,48 & 264,60 & 35,60 & - \\
\hline \multirow{2}{*}{$\begin{array}{c}\text { Val eur } \\
\text { énergétique }\end{array}$} & en cal. & 308,11 & 327.68 & 167,91 & 270,08 & 57,80 & - \\
\hline & en $U_{\text {. }}$. & 0,085 & 0,095 & 0,048 & 0,078 & 0,016 & - \\
\hline
\end{tabular}


d'orge constitue l'unité fourragère, et la valeur fourragère d'un aliment est la quantité d'orge qui produirait le même effet énergétique qu'un kilogramme de cet aliment ». Le système des unités fourragères danoises, d'après CRAPLET, est largement diffusé en France où il constitue la méthode officielle française (2).
A. VIALARD GOUDOU (8). Par la suite, les responsables de cette Société hippique saigonnaise s'adressèrent sur nos conseils à d'autres fournisseurs qui leur livrèrent un paddy de meilleure qualité. Ce dernier échantillon présentait - pour une humidité ramenée à 42,01 p. 100, taux d'humidité du premier échantillon - les

TABLEAU II - Composition chimique de divers fourrages

\begin{tabular}{|c|c|c|c|c|c|c|}
\hline & $\begin{array}{c}\text { Valeur } \\
\text { énergétique } \\
\left(\mathrm{U}_{.} \mathrm{F} .\right)\end{array}$ & $\begin{array}{c}\text { Cellulose } \\
(\mathrm{g})\end{array}$ & $\begin{array}{c}\text { Protides } \\
(\mathrm{g})\end{array}$ & $\begin{array}{c}\text { Glucides } \\
(\mathrm{g})\end{array}$ & $\begin{array}{c}\text { Calcium } \\
(\mathrm{g})\end{array}$ & $\begin{array}{c}\text { Phosphore } \\
(\mathrm{g})\end{array}$ \\
\hline Herbe verte SaIgon & 0,168 & 185 & 43 & 89 & 2,65 & 0,98 \\
Graminées jeunes & 0,180 & 63 & 51 & 138 & 1,00 & 0,90 \\
Graminées mares & 0,150 & 106 & 30 & 141 & 1,00 & 1,10 \\
Graminées_trème & 0,150 & 65 & 47 & 145 & 1,50 & 0,40 \\
\hline
\end{tabular}

Or, si l'on se réfère aux tables alimentaires de $M^{\text {me }}$ Lucie RANDOIN (4), on voit que 1 kilogramme de farine d'orge, soit 1 unitéfourragère équivaut à 3.440 calories. Cette relation nous a permis de convertir en $U$. F. les valeurs énergétiques tout d'abord exprimées en calories.

Connaissant d'une part la valeur énergétique et la composition chimique centésimale des 6 aliments consommés, et d'autre part les quantités ingérées par jour et par cheval, il nous a été facile d'établir - pour chaque aliment et ensuite pour l'ensemble de la ration journalière l'apport quotidien des éléments nutritifs (lipides, protides, glucides) ou de ballast (cellulose, silice) ainsi que l'apport des ions minéraux (calcium. phosphore, fer, sodium et potassium). Nous avons en outre calculé les bilans énergétiques en calories et en Unités Fourragères.

Dans le Tableau I, nous avons groupé en les rapportant à 100 grammes de produit les compositions chimiques et les valeurs énergétiques des 6 aliments constituant lo ration:

- Le riz corgo ef le mais, donnés aux chevaux, correspondent aux normes indiquées par Mme Lucie RANDOIN et A. VIALARD GOUDOU dans leurs tables alimentaires.

- Le paddy germé utilisé peut être considéré par contre comme de qualité inférieure, trop riche en silice et en cellulose, comparativement aux chiffres moyens proposés par AURIOL et pourcentages suivants: 10,16 de cellulose au lieu de 14,$60 ; 1,98$ de silice au lieu de 2,25, 39,18 de matières protéiques au lieu de 34,87 ; 0,054 U. F. pour 100 grammes au lieu de 0,048 .

- La mélasse de canne dsucre comme les échantillons en provenance du marché sud-vietnamien que nous avions antérieurement examinés, présente des teneurs élevées en sucre (sucres réducteurs 19,70 p. 100 - saccharose 46,45 p. $100)$ et en calcium. $(859,5 \mathrm{mg}$ pour $100 \mathrm{~g})$. De plus, la valeur du rapport $\mathrm{Ca} / \mathrm{P}$ de cette denrée doit retenir l'attention pour les raisons suivantes :

c) Les céréales amylacées - riz cargo, maïs, paddy germé - qui composent en majeure partie la ration de ces chevaux, sont caractérisées par un déséquilibre du rapport $C a / P$, résultant d'un fort excès de phosphore (valeur moyenne du rapport $\mathrm{Ca} / \mathrm{P}$ des céréales: 0.1$)$.

b) Le cheval exige un apport de calcium alimentaire plus grand que l'Homme (les taux de calcium sanguin sont respectivement de $100 \mathrm{mg}$ par litre chez l'Homme, et de $130 \mathrm{mg}$ par litre chez le Cheval). La valeur du rapport $\mathrm{Ca} / \mathrm{P}$ de la ration du cheval doit être comprise entre 1 ef 2 selon CRAPLET, ou voisine de 1,8 selon d'autres auteurs (3). Rappelons pour mémoire que chez l'homme adulte le calcium et le phosphore s'équilibrent convenablement lorsque le ropport $\mathrm{Ca} / \mathrm{P}$ est égal à 0,8 . 
TABLEAU III - APPORT GLOBAL ALIMENTAIPE ET ÉNERGETIOUE PAR CHEVAL ET PAR JOUR Avec l.a répartition par aljuerls couposant la ration jourmelière.

\begin{tabular}{|c|c|c|c|c|c|c|c|c|}
\hline & & $\begin{array}{l}\text { Riz cargo } \\
(3.500 \mathrm{~g})\end{array}$ & $\begin{array}{c}\text { Masa } \\
(600 \mathrm{~g})\end{array}$ & $\begin{array}{c}\text { Faddy gormé } \\
(1.650 \text { E) }\end{array}$ & $\begin{array}{l}\text { Mélasse } \\
\text { de canne } \\
(350 \mathrm{~g})\end{array}$ & $\begin{array}{c}\text { Herbe verte } \\
(5.000 \mathrm{~g})\end{array}$ & $\begin{array}{c}\text { Sel } \\
(20 \mathrm{~g})\end{array}$ & $\begin{array}{l}\text { Apport global } \\
\text { en grammes. }\end{array}$ \\
\hline \multicolumn{2}{|c|}{ Lipides (en g) } & 66,85 & 35,76 & 20,29 & 0 & 28,00 & 0 & 150,90 \\
\hline \multicolumn{2}{|c|}{ Protides (en g) } & 227,15 & 53,58 & 71,61 & 4,79 & 215,50 & 0 & 572,63 \\
\hline \multicolumn{2}{|c|}{ Glucides (en s) } & 2318,40 & 357,48 & 575,35 & 231,52 & $4,45,50$ & 0 & 3928,25 \\
\hline \multicolumn{2}{|c|}{$\begin{array}{c}\text { Cellulose et } \\
\text { matières de déchet } \\
\text { (en g) }\end{array}$} & 126,35 & 47,58 & 240,90 & - & 927,00 & 0 & 1341,83 \\
\hline \multicolumn{2}{|c|}{$\begin{array}{c}\text { Cendres minérales } \\
\text { (en g) }\end{array}$} & $42, \infty 0$ & 10,44 & 48,34 & 18,13 & 189,00 & 18,91 & 326,82 \\
\hline \multicolumn{2}{|c|}{ silice (en $g$ ) } & 4,06 & 0,44 & 37,12 & $1, \infty$ & 82,10 & 0 & 124,73 \\
\hline \multicolumn{2}{|c|}{ Phosphore (en g) } & 8,57 & 2,10 & 1,92 & 0,11 & 4,90 & 0 & $.17,60$ \\
\hline \multicolumn{2}{|c|}{ Calcium (en $g$ ) } & 0,47 & 0,07 & 0,32 & 3,01 & 13,20 & 0,04 & 17,12 \\
\hline \multicolumn{2}{|c|}{ Potassium (en g) } & 6,19 & 2,04 & 1,75 & 5,25 & 28,25 & 0 & 43,48 \\
\hline \multicolumn{2}{|c|}{ Sodium (en g) } & 1,08 & 0,24 & 0,25 & 0,57 & 1,60 & 6,81 & 10,55 \\
\hline \multicolumn{2}{|c|}{ Fer (en g) } & 0,09 & 0,02 & 0,09 & 0,06 & 0,52 & 0 & 0,78 \\
\hline \multirow{3}{*}{$\begin{array}{l}\text { Calories } \\
\text { dues } \\
\text { aux }\end{array}$} & lipides & 602 & 322 & 183 & 0 & 252 & 0 & 1.350 \\
\hline & protideo & 909 & 214 & 286 & 19 & 862 & 0 & 2.291 \\
\hline & glucides & 9.274 & 1.430 & 2.301 & 926 & 1.782 & 0 & 15.713 \\
\hline \multirow{2}{*}{\multicolumn{2}{|c|}{$\begin{array}{l}\text { Pouvoir } \\
\text { énergétique } \\
\text { total }\end{array}$ en cal. }} & 10.784 & 1.966 & 2.770 & 945 & 2.896 & 0 & 19.362 \\
\hline & & 3,13 & 0,57 & 0,80 & 0,27 & 0,84 & 0 & 5,62 \\
\hline
\end{tabular}

L'ingestion de mélasse (Ca/P: 28,65) par le cheval, même en petites quantités, permet de compenser de façon satisfaisante l'insuffisance en calcium de sa ration (Cf. Tableaux I et III).

- L'herbe verte fournie aux chevaux sans être excellente peut convenir : elle présente sensiblement la même valeur énergétique que les échantillons d'herbes types mentionnés par
CRAPLET. II en est de même pour la composition chimique, à noter toutefois des taux plus élevés en cellulose et en calcium, plus faibles en glucides, comme il ressort du tableau suivant (Résultats rapportés à 1 kilogramme d'herbe).

Dans le Tableau III, nous avons rassemblé les quantités de lipides, protides, glucides, cellulose, cendres minérales, silice, phosphore, calcium, 
sodium, potassium, fer et les valeurs énergétiques correspondantes (en calories et en unités fourragères) apportées par jour et par cheval, d'une part par chacun des 6 aliments indiqués et d'autre part par la ration globale.

\section{Caractéristiques de la ration}

En résumé, chaque cheval reçoit par jour : - 19.362 calories, soit 5,6 unités fourragères.

Ces calories proviennent pour $81,15 \%$ des glucides, pour $11,83 \%$ des protides, pour $7,01 \%$ des lipides.

- 572 grammes de protides.

- 780 milligrammes de fer.

$$
\begin{aligned}
& \text { - Rapport } \frac{\mathrm{Ca}}{\mathrm{P}}: \frac{17,12 \text { grammes }}{17,60 \text { grammes }}=0,97 \\
& \text { - Rapport } \frac{\mathrm{K}}{\mathrm{Na}}: \frac{43,48 \text { grammes }}{10,55 \text { grammes }}=4,12
\end{aligned}
$$

\section{Données théoriques}

de l'alimentation rationnelle du cheval

Elles sont difficiles à établir, car elles dépendent de l'age, du poids, des fonctions physiologiques (croissance, gestation et allaitement), de la nature du travail à effectuer et de l'utilisation du cheval (cheval de trait, d'attelage, de selle, de course...), de la race, des conditions climafiques et des saisons, sans parler des particularités individuelles propres à chaque cheval.

De là s'explique l'apparente non-concordance des chiffres fournis par les divers auteurs qui se sont préoccupés de ces questions.

Pour fixer les idées nous donnerons quelques exemples :

a) Un cheval de course, selon R. AMIOT (1), dont la ration à base d'avoine $(6 \mathrm{~kg})$, de mélasse $(2,5 \mathrm{~kg})$, de foin $(2,5 \mathrm{~kg})$, de mais $(1,5 \mathrm{~kg})$, de carottes $(0,5 \mathrm{~kg})$ et de féverolles $(0,1 \mathrm{~kg})$ est répartie en 4 repas à $5,10,15$ et 18 heures, reçoit par jour :

- 34.724 calories soit 10 unités fourragères

- 962 grammes de protides

$-39,43$ grammes de calcium et 30,95 grammes de phosphore soit $\mathrm{Ca} / \mathrm{P}: 1,27$

$-1.396,5$ milligrammes de fer.

b) Un cheval de gros trait de $600 \mathrm{~kg}$ (culture ou camionnage) selon R. Amiot (1), nourri d'avoine
$(9 \mathrm{~kg})$, de foin $(9 \mathrm{~kg})$ et de paille mélassée ( 5 litres), distribués en 3 fois le matin, le midi et le soir doit disposer par jour de :

- 43. 894 calories soit 12,7 unités fourragères

-1.617 grammes de protides

- 62,5 grammes de calcium et 50,5 grammes de phosphore, soit Ca/P: 1,24

- 2.774 milligrammes de fer.

c) Un cheval ou un mulet d'Europe en service dons les troupes du Sud-Indochinois, selon M. ROCHEFRETTE (7), avait avant 1948 une ration quotidienne à base de paddy $(4,4 \mathrm{~kg})$, d'herbe verte $(13 \mathrm{~kg})$, de paille de paddy $(4 \mathrm{~kg})$, de canne à sucre $(0,5 \mathrm{~kg})$, de sel $(20 \mathrm{~g})$, de carbonate de calcium $(7,5 \mathrm{~g})$ équivalant à :

- 17.750 calories soit 5,1 unités fourragères

- 643 grammes de protides

- 23,09 grammes de calcium et 16,35 grammes de phosphore soit $\mathrm{Ca} / \mathrm{P}: 1,41$

- 685 milligrammes de fer.

d) Un cheval de trait en Europe pesant 450 kilogrammes, selon CRAFI.ET (2) pour assurer ses besoins d'entretien $e^{\prime}$ de production doit recevoir une nourriture présentant les caractéristiques suivantes :

7,5 unités fourragères

450 grammes de protides

Rapport $\mathrm{Ca} / \mathrm{P}$ compris entre 1 et 2 .

Rapport $\mathrm{K} / \mathrm{Na}$ inférieur à 3.

\section{Discussion. Améliorations à apporter à la ration des chevaux de la société hippique saïgonnaise}

Les chiffres que nous avons trouvés s'accordent assez bien avec ceux rapportés par ROCHEFRETTE (chevaux originaires d'Europe en service dans les troupes du Sud Indochinois) et ne s'éloignent pas trop des données très générales indiquées par CRAPLET pour des chevaux de $450 \mathrm{~kg}$. Les 7,5 unités fourragères recommandées par cet auteur nous semblent excessives en région tropicale où l'animal comme l'homme n'a pas besoin de disposer d'un excès de calories pour lutter contre le froid. Par contre la valeur du rapport $\mathrm{Ca} / \mathrm{P}$ de la ration envisagée $(\mathrm{Ca} / \mathrm{P}$ : 0,97 ) révèle sans aucun doute une déficience en ion calcium. De plus le rapport potassiumsodium $(K / \mathrm{Na}: 4.12)$ indique une insuffisance 
d'ion sodium dans l'alimentation de ces chevaux.

A la lumière de tous ces résultats, et compte tenu de la physiologie particulière du cheval, « qui souffre le plus souvent de l'excès que du défaut de nourriture » (2) nous pensons que la ration alimentaire des chevaux de cette société hippique saïgonnaise pourrait être améliorée, si on la modifiait comme suit :

le matin

- 2 litres $1 / 2$ de riz cargo rouge soit 2.900 grammes

- 1 litre de maïs soit 600 grammes

- mélasse de canne à sucre 470 grammes (Ce poids correspond au contenu d'une boîte standard de lait concentré sucré) - 40 grammes de sel ordinaire.

le soir

- 2 litres $1 / 2$ de paddy germé de bonne qualité soit 1.375 grammes.

entre les 2 repas et principalement le soir :

- Herbe verte : 5 kilogrammes

- Eau : ad libitum.

Cette ration modifiée fournit par jour et par cheval :

- 17.651,63 calories, soit 5,1 unités fourragères. Ces calories proviennent pour $81,17 \%$ des glucides, pour $11,83 \%$ des protides, pour $6,98 \%$ des lipides.

- 552 grammes de protides.

- 712 milligrammes de fer.

- Rapport $\frac{\mathrm{Ca}}{\mathrm{P}}: \frac{21,48 \text { grammes }}{16,10 \text { grammes }}: 1,33$.

- Rapport $\frac{\mathrm{K}}{\mathrm{Na}}: \frac{43,93 \text { grammes }}{17,31 \text { grammes }}: 2,53$

Les quelques modifications apportées à la composition de la ration et à la répartition des aliments entre les repas du matin et du soir se justifient pour les raisons énumérées ci-après :

10 Par crainte d'une alimentation excessive, le pouvoir énergétique global a été légèrement réduit de 5,6 unités fourragères à 5,1 unités fourragères, de même que l'apport de protides a été réduit de 572 grammes à 552 grammes. Ces résultats ont été obtenus en diminuant d'un sixième les quantités de riz, cargo rouge et de paddy germé distribuées chaque jour.

20 La déficience en calcium de la ration a été en partie corrigée en augmentant d'un quart les fournitures de mélasse de canne. De cette façon, chaque cheval reçoit par jour 21,48 grammes de calcium au lieu de 17,12 grammes, et 16,10 grammes de phosphore au lieu de 17,60 grammes. Ces modifications se traduisent par une valeur du rapport $\mathrm{Ca} / \mathrm{p}$ beaucoup plus satisfaisante $\mathrm{Ca} / \mathrm{p}=1,33$ au lieu de 0,97, valeur nettement insuffisantc.

30 Le fait de doubler l'apport de sel ordinaire - 40 grammes au lieu de 20 grammes par jour - permet de ramener la valeur du rapport $\mathrm{K} / \mathrm{Na}$ au-dessous de 3, comme le recommande CRAPLET (2).

40 Les aliments à pouvoir énergétique élevé et facilement assimilables (mélasse de canne, maïs, riz cargo rouge) sont distribués au repas du matin, alors que les aliments riches en cellulose, moins rapidement digérés sont donnés l'après-midi (herbeverte) et le soir (paddy germé).

50 Pour compléter les bienfaits d'une alimentation rationnelle, on devra aussi observer les règles d'hygiène classiques: hygiène corporelle du cheval, surveillance permanente de la dentition, hygiène des étables. II ne faudra pas non plus négliger d'exposer les chevaux au soleil : la synthèse in vivo de la vitamine $D$ est en effet réalisée par irradiation aux rayons solaires ultra-violets. Non seulement cette vitamine assure la fixation du calcium, mais encore elle régularise l'équilibre phosphocalcique de l'organisme. Enfin, lorsque les chevaux auront à disputer les épreuves d'un concours hippique, on pourra adjoindre à leur nourrifure 500 grammes de carottes râpées qui, riches en provitamine $A$, augmenteront leur acuité visuelle.

Institut Pasteur de Saïgon. 


\section{RESUMÉ}

Après avoir déterminé la composition chimique et la valeur calorifique des aliments - paddy germé, maïs, mélasse de canne à sucre, riz cargo rouge, sel ordinaire et herbe verte - constituant la ration des chevaux de selle d'une Société Hippique à Saïgon, nous avons calculé l'apport global, par cheval et par jour, de chacun des principes nutritifs et énergétiques, en particulier le pouvoir calorifique, l'apport en protides, en calcium et phosphore, en potassium et sodium, et en fer.

La comparaison des chiffres obtenus avec ceux publićs par d'autres autcurs nous a conduit à modifier quelque peu la composition de la ration et la répartition des 6 aliments précités entre les repas du matin et du soir.

La nouvelle ration proposée correspond, par jour et par cheval (poids moyen $450 \mathrm{~kg}$ ), à 5,1 Unités Fourragères soit 17.650 calories, à 550 grammes de protides, à 21,5 grammes de calcium, à $710 \mathrm{mil}$ ligrammes de fer, et présente des rapports $\mathrm{Ca} / \mathrm{P}$ et $\mathrm{K} / \mathrm{Na}$ respectivement égaux à 1,33 et 2,53.

Les aliments sont ingérés le matin à l'exception de l'herbe verte (l'après-midi) et du paddy germé (le soir) qui, par suite de leur teneur en cellulose, ne sont que lentement digérés.

\section{SUMMARY}

\section{Study on the diet of the horses of a riding school in Saigon}

Having analysed the chemical composition and the calorimetric value of the feeds (germinated rice, Maize, sugar cane molasses, red cargo rice, common salt and green forage) of which the diet of the riding horses were composed, the total daily consumption in nutrients and energy, in particular the calorific value, the proteins, calcium phosphorus and potassium sodium ratios and of iron, was assessed.

A comparison of the resulting figures with those presented by other workers led the author to compose a slightly different diet and to alter the feeding times.

The new proposed daily ration meets the requirements of a $1.000 \mathrm{lbs}$ horse. e. i., 5.1 feeding units $=17,650$ calories, made up from $550 \mathrm{grms}$ of proteins, 21,5 grams of calcium and $710 \mathrm{mgrms}$ of iron. Its $\mathrm{Ca} / \mathrm{P}$ and $\mathrm{K} / \mathrm{Na}$ ratios are respectively 1.33 and 2.53 .

The highly digestible foodstuffs are given earlier in the day. The greenstuffs are fed in the afternoon and the germinated rice in the evening, their higher fibre content being more slowly digested.

\section{RESUMEN}

Estudio de la ración alimenticia de los caballos de una Sociedad hípica de Saigón.

Después de haber determinado la composición química y el valor en calorias de los alimentos - arroz germinado, maíz, melaza de can̂na de azucar, arroz en cáscara rojo, sal ordinaria y hierba verde - que constituyen la ración de los caballos de silla de una Sociedad hípica de Saigón, hemos calculado la cantidad de cada uno de los principios nutritivos y energéticos, en particular calorias, prótidos, calcio y fósforo, potasio y sodio, hierro, que cada animal recibe por dia.

La comparación de las cifras obtenidas con las que ofros autores han publicado, nos ha llevado a modificar un poco la composición de la ración y el reparto de los seis alimentos citados entre las comidas de la mañana y de la tarde.

La nueva racion propuesta corresponde, por dia y animal peso medio de $450 \mathrm{kgs}$ ), à 5,1 unidades forrajeras, que equivalen a 17.650 calorias, 550 grs. de protidos ; 21,5 grs. de calcio ; 710 mgrs. de hierro ; cociente calcio-fósforo $=1,33$ y cociente potasio-sodio $=2,53$.

Los alimentos se suministran por la mañana a excepción de la hierba verde (medio día) y del arroz germinado (la tarde) que, por su alto contenido en celulosa, son digeridos lentamente. 


\section{BIBLIOGRAPHIE}

1. AMIOT (R.). - Le Cheval, 1 re éd., P. U.F. Ed., Paris 1949.

2. CRAPLET (C.). - L'Alimentation du Bétail, 1 re éd., Vigot éd., Paris 1950.

3. HUARD (M.). - Inst. Pasteur vét. Nhatrang (Viet-Nam) Communication personnelle.

4. RANDOIN (L.), CAUSERET (J.) et LEGALLIC (P.).- - Table de composition des aliments, $2^{e}$ éd. : Lanore Edit., Paris, 1947.

5. RENAULT (J.). - Le tétraphénylborure de sodium comme réactif analytique. Mises au point de chimie analytique pure et appliquée. Masson Edit., Paris, 1958, 6e série, p. 110-125.
6. RIBEREAU-GAYON (J.) et PEYNAUD (E.). Dosage du sodium dans les vins. Analyse ef contrôle des vins, $2 \mathrm{e}$ édit., Béranger Edit., Paris-Liège, 1958, p. 190-191.

7. ROCHEFRETTE (M.). - Hygiène et pathologie des équipages dans le Sud-Indochinois. Rev. vét. militaire, 1948, 3, p. 26-66.

8. VIALARD GOULOU (A.). - Recherche sur quelques plantes alimentaires du Sud VietNam et de l'Asie Tropicale. Composition chimique. Valeur nutritive. Emploi dans l'alimentation Th. Doct. ès Sc., Bordeaux, 1956. 